\title{
Chronic Localized Intravascular Coagulation in a Case of Sporadic Multifocal Venous Malformations with Phleboliths
}

\author{
${ }^{1}$ Ajeet K Chaurasia, ${ }^{2}$ Poonam Gupta, ${ }^{3}$ Naincy Purwar
}

\begin{abstract}
Aim: The aim of this article is to report a case of chronic localized intravascular coagulation in widespread sporadic multifocal venous malformations (VMs) associated with phleboliths, presenting with pathological femur fracture with very high D-dimer level and deranged coagulation profile hampering the surgical management of the patient.
\end{abstract}

Background: Disseminated intravascular coagulation (DIC) is a well-known cause of raised D-dimer. It leads to derangement of coagulation profile with very poor management outcome and needs intensive care. A very high level of D-dimer present in localized intravascular coagulopathy (LIC) can be misleading to diagnosis of DIC. Localized intravascular coagulopathy is seen in few VMs and they show abnormally high D-dimer levels. Venous malformations are the most frequent slow-flow vascular malformations referred to specialized centers. Most of the VMs are sporadic, and unifocal, while $1 \%$ are multifocal VMs. Multifocal sporadic VMs are rare and associated with phleboliths. High D-dimer is associated with various types of VMs like cutaneomucosal VMs and capillarovenous malformations. We reported the case of a 22-year-old female with congenital multifocal VMs presenting with fracture shaft of left femur on trivial trauma with deranged coagulation profile as prolonged prothrombin time, activated partial thromboplastin time, thrombocytopenia, and persistently high D-dimer level along with the presence of multiple phleboliths.

Review: In the view of deranged coagulation profile with raised D-dimer level in presence of trauma, patient was initially managed as a case of DIC and her surgical procedure for fracture femur was delayed for the correction of her coagulation abnormality. She was transfused 16 units of fresh frozen plasma, 18 units of platelets, and 3 units of packed red blood cells, but her D-dimer level remained high. Persistently raised D-dimer level without any hemorrhagic manifestation along with the presence of multiple phleboliths in his X-rays clinched the diagnosis of LIC. Low molecular weight heparin was started and after stabilization of coagulation profile, the patient got shifted to orthopedics department for the management of fracture femur.

${ }^{1}$ Assistant Professor, ${ }^{2}$ Associate Professor, ${ }^{3}$ Junior Resident

${ }^{1-3}$ Department of Medicine, Moti Lal Nehru Medical College Allahabad, Uttar Pradesh, India

Corresponding Author: Poonam Gupta, Associate Professor Department of Medicine, Moti Lal Nehru Medical College Allahabad, Uttar Pradesh, India, Phone: +919198437676 e-mail: poonam_gupta16@yahoo.com
Conclusion: This case describes a unique presentation of sporadic multifocal VMs as coagulation abnormality mimicking DIC. It emphasizes prompt diagnosis and workup when multiple VMs are present to prevent morbidity during surgical excision secondary to intravascular coagulopathy.

Clinical significance: Multifocal VMs have been associated with an increased risk of spontaneous thrombosis and thrombolysis because of stasis of blood, a condition termed LIC. Severe LIC has potential to progress to DIC during surgical excision so this mandates preoperative evaluation.

Keywords: Localized intravascular coagulopathy, Phleboliths, Venous malformations.

How to cite this article: Chaurasia AK, Gupta P, Purwar N. Chronic Localized Intravascular Coagulation in a Case of Sporadic Multifocal Venous Malformations with Phleboliths. J Postgrad Med Edu Res 2017;51(3):144-149.

Source of support: Nil

Conflict of interest: None

\section{BACKGROUND}

Multiple venous malformations (VMs) pose some of the difficult challenges in the practice of medicine today. The clinical presentations are extremely wide and range from asymptomatic birthmarks to life-threatening hemorrhages. Because of the rarity of these lesions, experience in their diagnosis and management by clinicians is limited. This augments the enormity of the problem and can lead to misdiagnoses, inadequate treatment, high complication rates, and poor patient outcomes. Venous malformations are dilated venous, capillarovenous, and lymphatic channels with weak walls. Patients with VMs may have raised D-dimer (Table 1). Raised D-dimer level is associated with three types of malformations: ${ }^{1,2}$ sporadic venous (unifocal, multifocal) malformation; capillarovenous malformation; Klippel-Trenaunay syndrome. Disseminated intravascular coagulation (DIC) is a well-known cause of raised D-dimer and leads to derangement of coagulation profile and has poor management outcome. ${ }^{3}$ But the high level of D-dimer seen in localized intravascular coagulopathy (LIC) can lead to misdiagnosis of DIC. Localized intravascular coagulopathy is seen as abnormally high D-dimer in few VMs. ${ }^{4}$ Phleboliths are seen only in cases of sporadic VMs. Venous malformations are slow-flow 


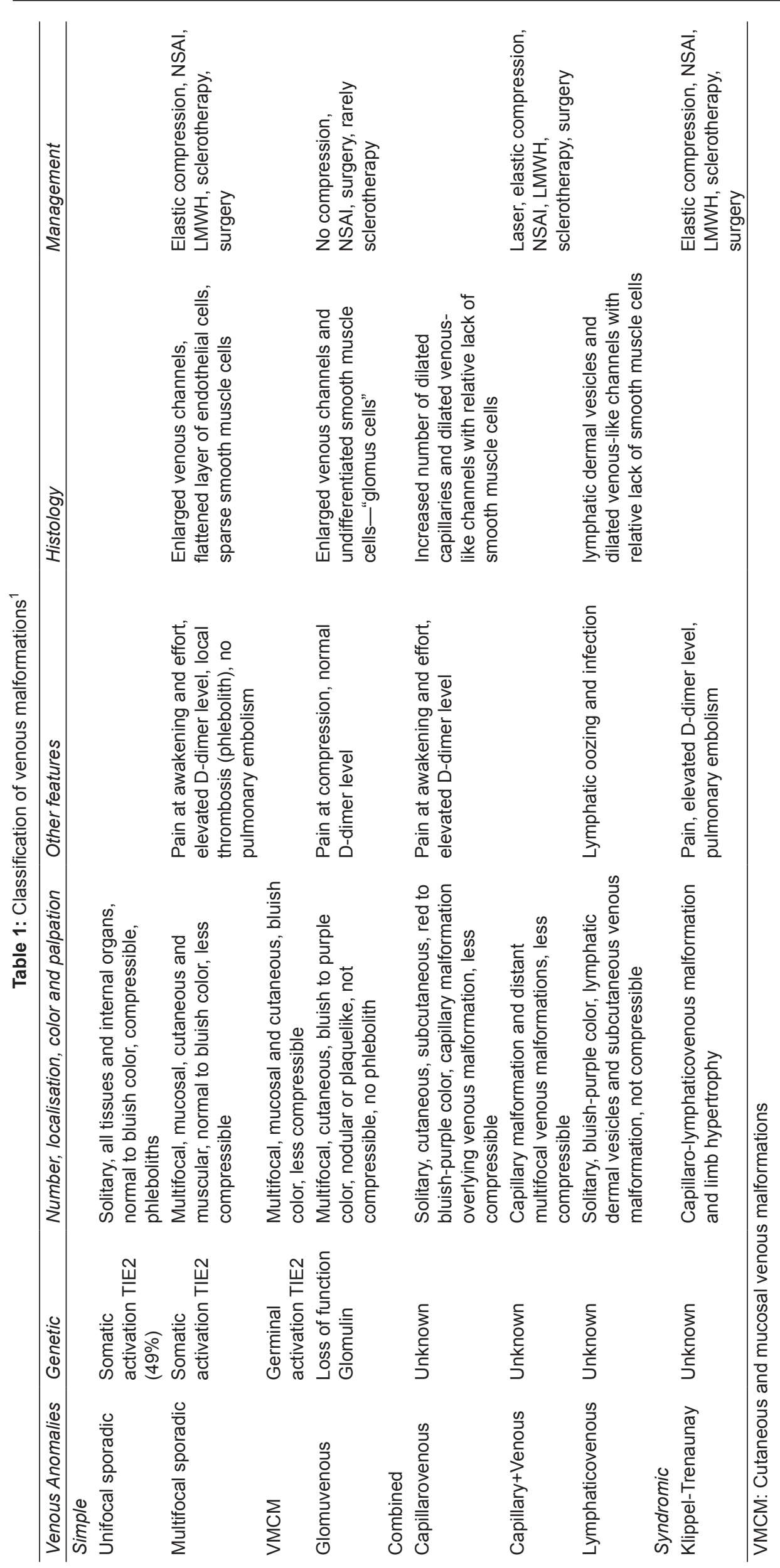


vascular malformations present at birth, and LIC causes pain and thrombosis within a lesion and severe bleeding during surgical procedures. ${ }^{5,6}$ Klippel-Trenaunay syndrome is also a cause of raised D-dimer levels and it is a triad of hemangiomas, varicose vein, and hemihypertrophy of the limb. ${ }^{2}$

\section{CASE REPORT}

A 22-year-old female having multiple congenital VMs at lateral canthus of right eye, left parotid gland, anterolateral aspect of right knee joint, left gluteal region, left knee joint anteriorly, abdominal wall, inferior angle of right scapula, dorsum of left foot, and sole of left foot (Figs 1 to 9) presented with fracture of shaft of left femur on trivial trauma. During her management at the primary care center by attending surgeon, she had excessive bleeding on the incision, so she was referred to our tertiary care center. On examination, the patient was found to be severely anemic. She had VMs as described and these VMs were partially compressible and painful. On deep palpation, phleboliths were felt. At presentation, her hemoglobin was $3.9 \mathrm{gm} / \mathrm{dL}$, platelet count $70,000 / \mathrm{mm}^{3}$, prothrombin

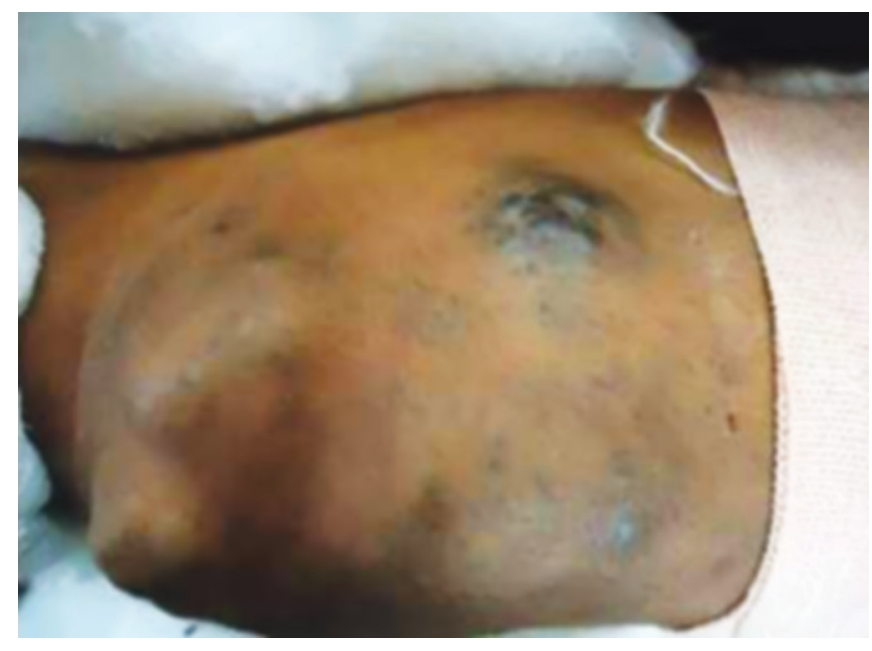

Fig. 1: Venous malformations around left knee joint

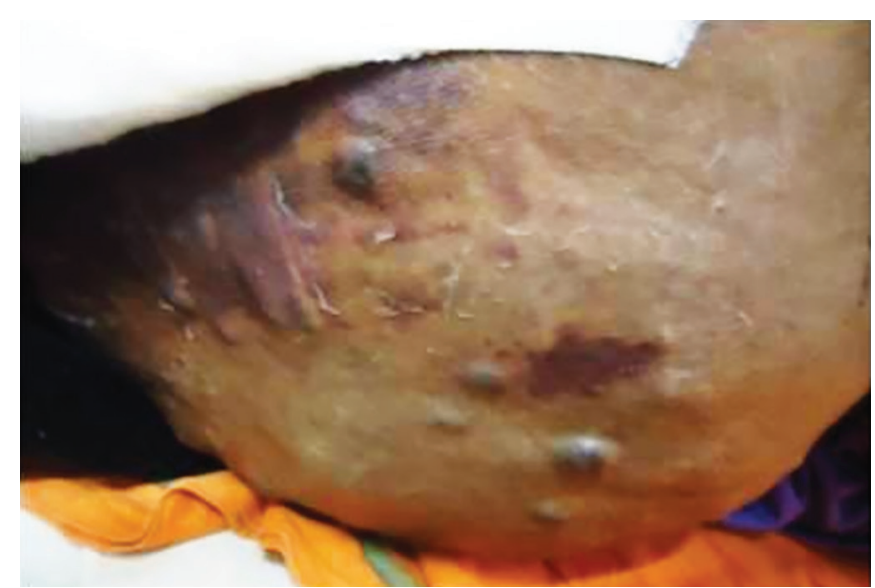

Fig. 3: Venous malformations at left buttock time (PT) 21.8 seconds, international normalized ratio 1.77 , activated partial thromboplastin time (aPTT) 43.6 seconds, D-dimer $1102.0 \mathrm{ng} / \mathrm{mL}$, serum bilirubin $0.52 \mathrm{mg} / \mathrm{dL}, \mathrm{C}$-reactive protein $6 \mathrm{mg} / \mathrm{L}$, plasma fibrinogen $170.0 \mathrm{mg} / \mathrm{dL}$, and serum antinuclear antibodies 5.74 units with normal liver and kidney function tests. The X-ray of left thigh showed displaced fracture femur shaft with multiple 2-5 mm circular, well-defined calcifications suggestive of phleboliths over thigh and pelvic soft tissue region (Fig. 10). Color Doppler study of left lower limb confirmed the presence of slow flow VMs and phleboliths. Color Doppler ultrasonography of left parotid region showed $20 \times 27 \times 35$ $\mathrm{mm}$ partially compressible multiloculated hypoechoic cystic intraparenchymal lesion in the superficial compartment of parotid gland suggestive of slow-flow VM. Conformational magnetic resonance imaging for the presence of VMs in internal organs was not done because of the metallic bone traction in situ.

\section{DISCUSSION}

Vascular anomalies are divided into two groups: Vascular tumors and vascular malformations. Hemangioma is

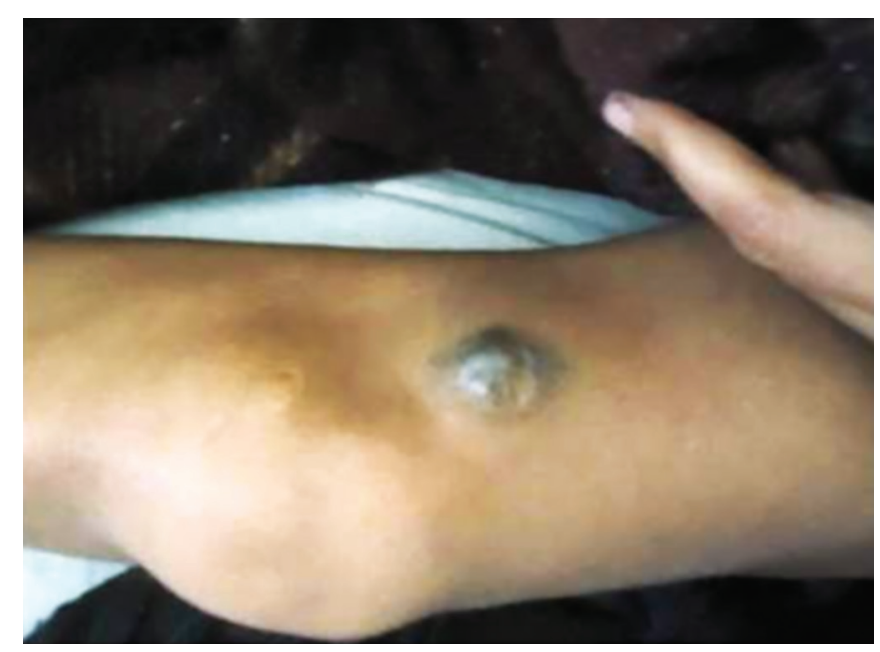

Fig. 2: Venous malformations above right knee joint

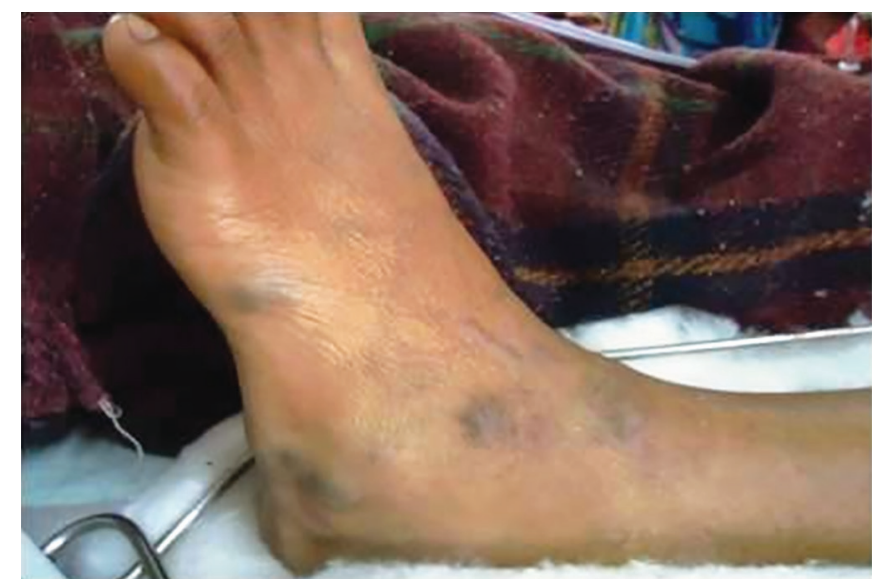

Fig. 4: Venous malformations near left ankle 


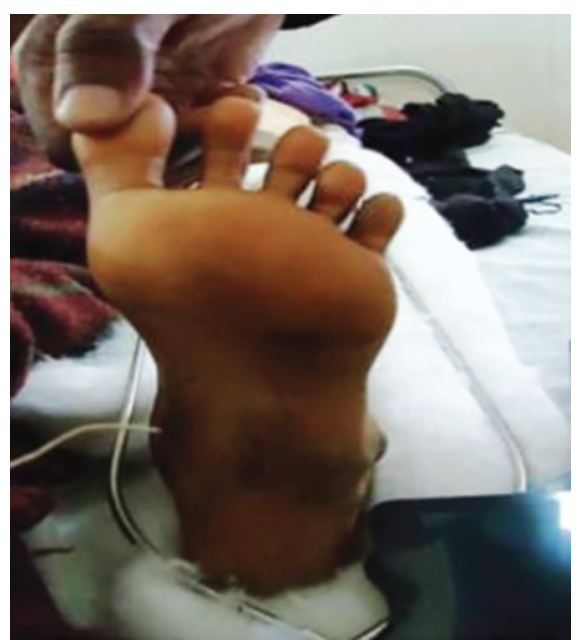

Fig. 5: Venous malformations on sole of left foot

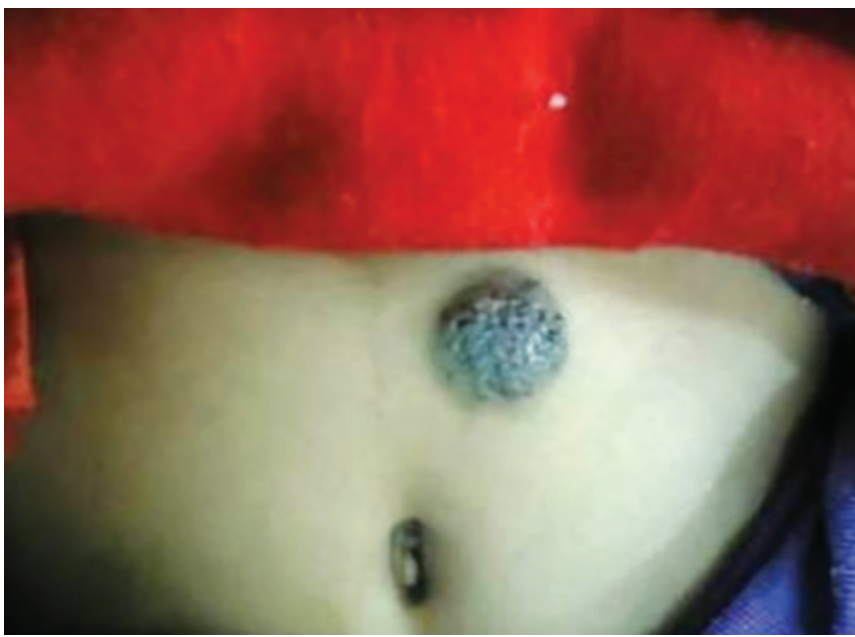

Fig. 7: Venous malformations over abdomen

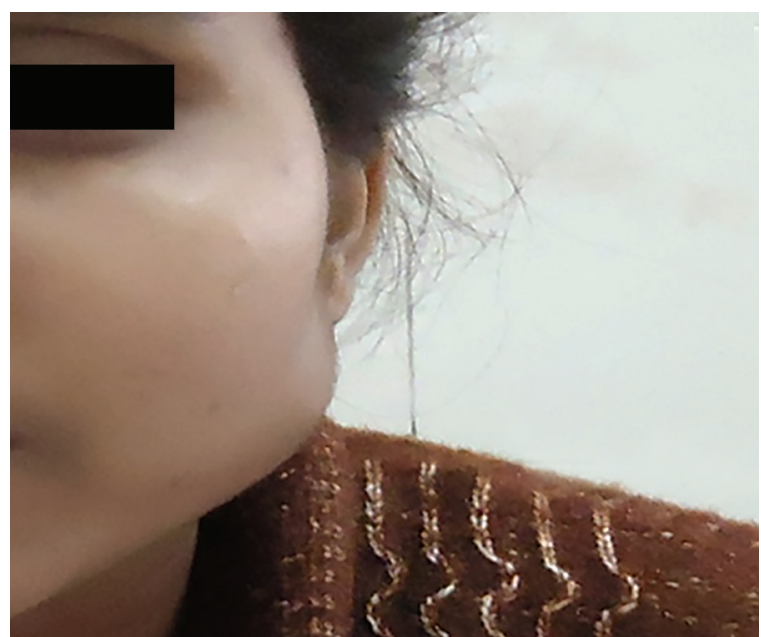

Fig. 9: Venous malformations-left parotid gland

the most common vascular tumor that usually appears postnatally. It exhibits rapid growth due to cellular proliferation, followed by inevitable involution. Hemangiomas have high-velocity flow in multiple vascular channels. On the contrary, vascular malformations are present

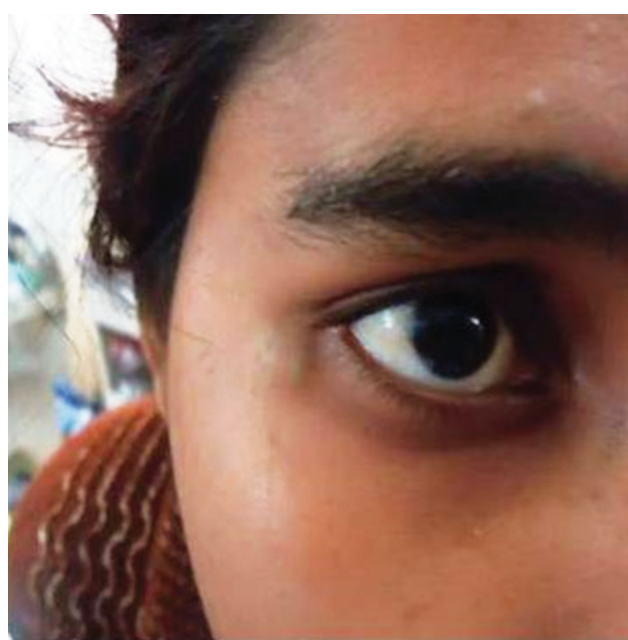

Fig. 6: Venous malformations-outer canthus of right eye

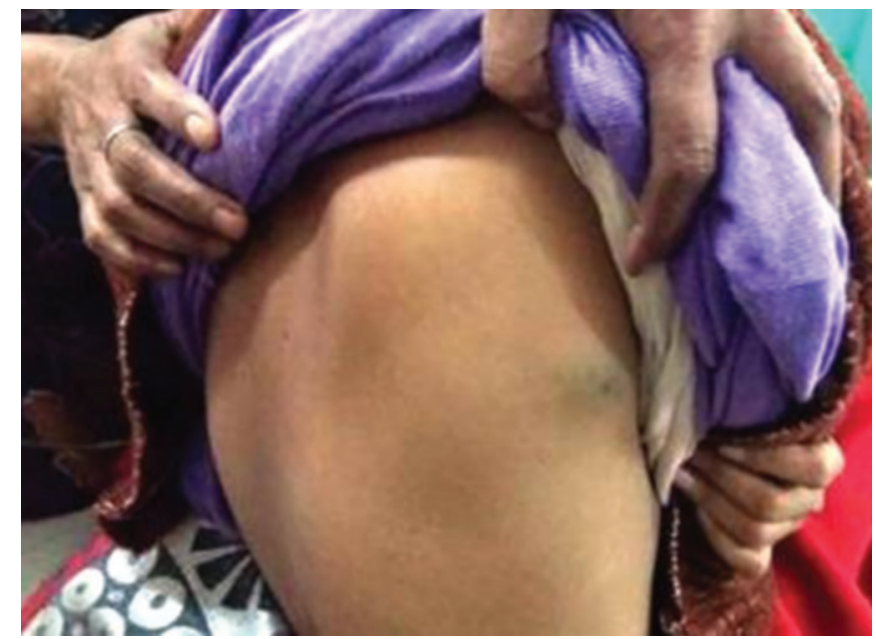

Fig. 8: Venous malformations near inferior angle of right scapula

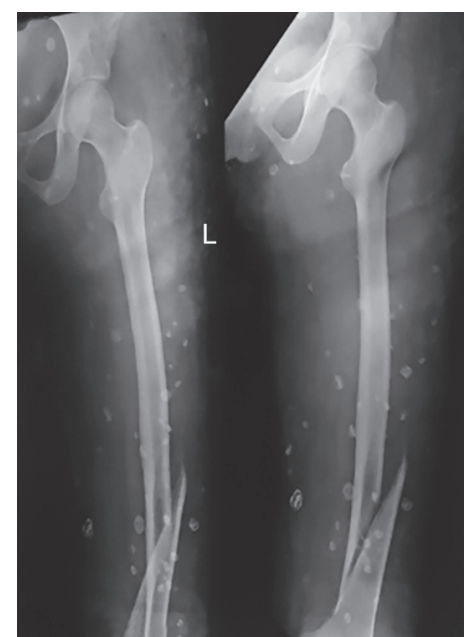

Fig. 10: Multiple phleboliths: X-ray left femur anteroposterior view

since birth and grow proportionally with the growth of patient. They are subdivided according to the affected vessel type into capillary, venous, arterial, and lymphatic malformations. They are slow-flow or fast-flow lesions. When malformations affect more than one vessel type, 
Flow Chart 1: Classification system of the International Society for the Study of Vascular Anomalies (1996). ${ }^{7}$ AVM: Arteriovenous malformation; CM: Capillary malformation; LM: Lymphatic malformation

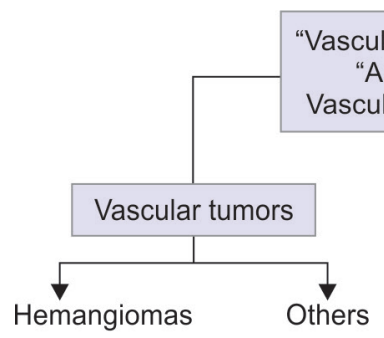

cular birthmarks

"Angiomas"

Vascular anomalies

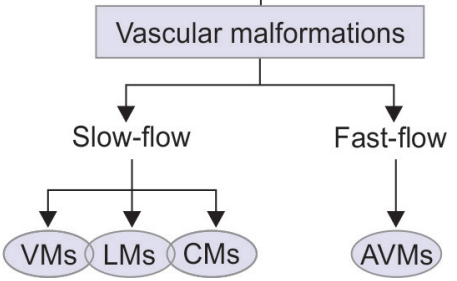

the combined lesions are named according to the affected vessel, e.g., capillarovenous and capillaro-lymphaticoVM (Flow Chart 1). Venous malformation can also occur in Klippel-Trenaunay syndrome (capillaro-lymphaticoVM with limb hypertrophy) and Maffucci syndrome (multiple endochondromas associated with multiple hemangioendothelioma and high incidence of malignancy). ${ }^{8,9}$ Our patient presented with multiple congenital vascular malformation present at lateral canthus of right eye, left parotid gland, anterolateral aspect of right knee joint, left gluteal region, left knee joint anteriorly, abdominal wall, inferior angle of right scapula, dorsum of left foot, and sole of left foot along with slow-flow VM present in parotid. The screening for the presence of VMs in internal organs could not be done. The presence of phleboliths favors VM, which was evident in soft-tissue X-ray of our patient. Due to blood stagnation, thrombosis can occur, leading to phlebolith formation. ${ }^{10,11}$ Multiple VMs are more common in females than males and more on left side of the body. We excluded Klippel-Trenaunay syndrome because there was no evidence of limb hemihypertrophy. D-dimer is the degradation product of cross-linked fibrin; therefore, it reflects ongoing activation of the hemostatic system. Elevated D-dimer levels reflect ongoing activation of the hemostatic and thrombolytic system, providing clinical utility in the diagnosis of DIC, deep vein thrombosis, and evaluation of thrombus formation. Additionally, D-dimer levels may be elevated in the setting of pregnancy, inflammation, malignancy, trauma, postsurgical treatment, and liver disease. It has been seen that higher VM severity scores (as in Table 2$)^{7}$ are associated with more severe LIC and bone fracture. ${ }^{12}$ In the view of deranged coagulation profile with raised D-dimer level in the presence of trauma, the patient was initially managed as a case of DIC and her surgery for fracture femur could not be done initially. Color Doppler of lower limb was done to rule out the presence of DVT as a cause of raised D-dimer. She was transfused 16 units of fresh frozen plasma, 18 units of platelets, and 3 units of
Table 2: Severity scoring system for superficial and deep VMs of the limbs and trunk ${ }^{7}$

\begin{tabular}{ll}
\hline Scoring Method & Criteria \\
\hline Each anatomic location is scored & Upper extremity, lower \\
as involved based on clinical & extremity, trunk, genitalia, \\
observation. One point is awarded & visceral location
\end{tabular}
for each location

Each type of tissue is scored as involved based on clinical and

Skin, subcutis, muscle, bone, joint magnetic resonance imaging evaluation. One point is awarded for each type of tissue

Total points

Minimum severity score 10

2 (1 location +1 tissue type involved)

packed red blood cells, but persistently raised D-dimer level and deranged coagulation profile like thrombocytopenia, raised PT and aPTT without any hemorrhagic manifestation along with presence of multiple phleboliths clinched the diagnosis of LIC. D-dimer is also raised in capillarovenous malformations but they are not associated with phleboliths and hence, were excluded. Lowmolecular-weight heparin was started after the diagnosis of LIC and patient was shifted to the orthopedic department for the definitive management of fracture femur after stabilization of patient coagulation profile.

\section{CONCLUSION}

Venous malformations are associated with spontaneous thrombosis and thrombolysis. This is witnessed by the presence of phleboliths and elevated D-dimer levels $(>0.5 \mu \mathrm{g} / \mathrm{mL})$. This phenomenon is named LIC. ${ }^{2} \mathrm{D}$-dimer levels are often very high $>1.0 \mu \mathrm{g} / \mathrm{mL}$ in $25 \%$ cases even if these otherwise healthy patients do not have underlying conditions that increase D-dimer levels. An LIC is usually well-tolerated during everyday life. However, a few patients are at risk of potential aggravation of LIC to DIC with dramatic bleeding during a surgical excision, and marked consumption of platelets, coagulation factors, and fibrinogen. ${ }^{4}$ Therefore, measurement of D-dimer levels is mandatory in the management of VMs. ${ }^{13}$ Venous malformation is not the only disease that can increase D-dimer levels highly and persistently in otherwise healthy patients, other conditions like glomerulonephritis, chronic rejection of renal allograft, and normal pregnancy may also lead to chronic LIC. ${ }^{14}$ Elevated D-dimer levels in vascular anomalies suggest a venous component in $96.5 \%$ of patients. This can help differentiate glomuvenous malformations from other multifocal venous lesions. It can also detect a venous component in combined and syndromic malformations. Low-molecular-weight heparin is used to relieve pain caused by LIC and to prevent decompensation of severe LIC to DIC. ${ }^{4}$ 


\section{CLINICAL SIGNIFICANCE}

1. Sporadic multifocal VMs are slow-flow vascular anomalies with wide clinical manifestations. We report a rare case of sporadic multifocal VM with raised D-dimer levels with phleboliths and severe anemia.

2. Severe coagulopathies, such as LIC and DIC are associated with multifocal VM, especially when the D-dimer level is elevated.

3. Anticipating the increased risk of severe coagulopathy, a thorough preoperative assessment for coagulation profile and preventive treatment with heparin as necessary before any surgical intervention is determined.

\section{ACKNOWLEDGMENT}

Authors are highly grateful to their senior librarian, $\mathrm{Mr} \mathrm{KP}$ Singh at MLN Medical College, Allahabad, for helping in getting access to various journal articles and books.

\section{REFERENCES}

1. Dompmartin A, Vikkula M, Boon LM. Venous Malformation: update on etiopathogenesis, diagnosis \& management. Phlebology 2010;25(5):224-235.

2. Lee BB, Laredo J, Lee TS, Huh S, Neville R. Terminology and classification of congenital vascular malformations. Phlebology 2007;22(6):249-252.

3. Venugopal A. Disseminated intravascular coagulation. Indian J Anaesth 2014 Sep;58(5):603-608.

4. Dompmartin A, Acher A, Thibon P, Tourbach S, Hermans C, Deneys V, Pocock B, Lequerrec A, Labbé D, Barrellier MT, et al. Association of localized intravascular coagulopathy with venous malformations. Arch Dermatol 2008 Jul;144(7):873-877.
5. Moake J. Disseminated Intravascular Coagulation (DIC). Merck Manual; 2013.

6. Halaby R, Popma CJ,Cohen A, Chi G, Zacarkim MR, Romero G, Goldhaber SZ, Hull R, Hernandez A, Mentz R, et al. D-Dimer elevation and adverse outcomes. J Thromb Thrombolysis 2015 Jan;39(1):55-59.

7. Mazoyer E, Enjolras O, Bisdorff A, Perdu J, Wassef M, Drouet J. Coagulation disorders in patients with venous malformation of the limbs and trunk: a case series of 118 patients. Arch Dermatol 2008 Jul;144(7):861-867.

8. Nosher JL, Murillo PG, Liszewski M, Gendel V, Gribbin CE. Vascular anomalies: a pictorial review of nomenclature, diagnosis and treatment. World J Radiol 2014 Sep 28;6(9):677-692.

9. Chava VR, Shankar AN, Vemanna NS, Cholleti SK. Multiple venous malformations with phleboliths: radiologicalpathological correlation. J Clin Imaging Sci 2013 Dec 31;3(Suppl 1):13.

10. Hein KD, Mulliken JB, Kozakewich HP, Upton J, Burrows PE. Venous malformations of skeletal muscle. Plast Reconstr Surg 2002 Dec;110(7):1625-1635.

11. Hermans C, Dessomine B, Lambert C, Deneys V. Venous malformation and coagulopathy. Ann Chir Plast Esthet 2006 Aug-Oct;51(4-5):388-393.

12. Mazoyer E, Enjolras O, Laurian C, Houdart E, Drouet L. Coagulation abnormalities associated with extensive venous malformations of the limbs: differentiation from KasabachMerritt syndrome. Clin Lab Haematol 2002 Aug;(24):243-251.

13. Dompmartin A, Ballieux F, Thibon P, Lequerrec A, Hermans C, Clapuyt P, Barrellier MT, Hammer F, Labbé D, Vikkula M, et al. Elevated D-dimer level is diagnostic for venous malformations. Arch Dermatol 2009 Nov;145(11):1239-1244.

14. Schmer G, Strandjord PE, editor. Intravascular coagulationacute and chronic-disseminated and local. In: Coagulation: Current research and clinical applications. Academic Press; 1973. p. 58. 